Bull. Austral. Math. Soc.

VoL. 53 (1995) [293-297]

\title{
A NOTE ON FRAME STARTERS
}

\author{
Cheng-De Wang
}

\begin{abstract}
We construct frame starters in $Z_{2 n}-\{0, n\}$, for $n \equiv 0,1 \bmod 4$, where $Z_{2 n}$ denotes the cyclic group of order $2 n$. We also construct left frame starters in $Q_{2 n}-\left\{e, \alpha^{n}\right\}$, where $Q_{2 n}$ is the dicyclic group of order $4 n$ and $\alpha^{n}$ is the unique element of order 2 in $Q_{2 n}$.
\end{abstract}

\section{INTRODUCTION}

In this paper we provide specific constructions of frame starters in cyclic groups of even order and in dicyclic groups. This work is motivated by two different aspects of previous investigations in combinatorics.

The concept of a near complete mapping and a near orthomorphism in a group was introduced in [2] as follows. A finite group $G$ is said to have a near complete mapping $\theta$ if $\theta$ is a bijection from $G-\{h\}$ to $G-\{e\}$ where $h$ is some fixed element of $G$ and $e$ is the identity element of $G$, in such a way that the map $\varphi: g \rightarrow g \theta(g)$ is another bijection from $G-\{h\}$ to $G-\{e\}$. The map $\varphi$ is called a near orthomorphism from $G-\{h\}$ to $G-\{e\}$. Groups which have no orthomorphisms may have near orthomorphisms. It was shown in [2] that a group $G$ which has either an orthomorphism or a near orthomorphism can be used to construct left neofields.

If $G$ is a group with a unique element $h$ of order 2 , a near orthomorphism from $G-\{h\}$ to $G-\{e\}$ was called a special near orthomorphism of $G$ in [4]. A special near orthomorphism of $G$ can be regarded as a bijection $\varphi$ from $G-\{e, h\}$ to itself such that $x^{-1} \varphi(x)=y^{-1} \varphi(y)$ if and only if $x=y$. Special near orthomorphisms in cyclic groups of even order have connections with the investigation of the admissibility of linear groups.

On the other hand, the concept of a starter was generalised to the concept of a frame starter in [1]. Actually, in the definitions of starters or frame starters, we need not assume the commutative law in the related group [3]. Let $G$ be a group of order $n$ and $H$ be a subgroup in $G$ of order $m$ such that $n-m$ is even. A left frame starter in $G-H$ is a set of unordered pairs

$$
S=\left\{\left(s_{i}, t_{i}\right): 1 \leqslant i \leqslant(n-m) / 2\right\}
$$

Received 14 June 1995

Copyright Clearance Centre, Inc. Serial-fee code: 0004-9729/95 \$A2.00+0.00. 
such that the following two properties are satisfied

(1) $\left\{s_{i}: 1 \leqslant i \leqslant(n-m) / 2\right\} \cup\left\{t_{i}: 1 \leqslant i \leqslant(n-m) / 2\right\}=G-H$

(2) $\left\{s_{i}^{-1} t_{i}, t_{i}^{-1} s_{i}: 1 \leqslant i \leqslant(n-m) / 2\right\}=G-H$.

If we use $s_{i} t_{i}^{-1}$ and $t_{i} s_{i}^{-1}$ in (2), then we get a right frame starter. Notice that $\left\{\left(s_{i}, t_{i}\right): 1 \leqslant i \leqslant(n-m) / 2\right\}$ is a left frame starter if and only if $\left\{\left(s_{i}^{-1}, t_{i}^{-1}\right): 1 \leqslant i \leqslant\right.$ $(n-m) / 2\}$ is a right frame starter. In Abelian groups there is no difference between left and right starters. The most useful technique for direct construction of Room squares has been the starter-adder method. Room squares were generalised to Room frames [1] and it was shown that frame starters were very useful in the construction of Room frames [1].

We can see that a left frame starter in $G-\{e, h\}$, where $G$ is a group having a unique element $h$ of order 2 , determines a special near orthomorphism $\varphi$ which is the product of disjoint cycles of length 2, where $\varphi\left(s_{i}\right)=t_{i}$ and $\varphi\left(t_{i}\right)=s_{i}$ for all pairs in the frame starter. It was proved in [4] that every Abelian group $G$ having a cyclic Sylow 2 -group of order greater than 6 has a special near orthomorphism. On the contrary, it was stated that there were no frame starters in $G-H$ if $n / m \equiv 2$ or $3 \bmod 4$, where $G$ is an Abelian group of order $n$ and $H$ is a subgroup of order $m$. Hence there are no frame starters in $Z_{2 n}-\{0, n\}$ if $n \equiv 2$ or $3 \bmod 4$. This fact is also in contrast to the fact that for each group $G$ of odd order and any subgroup $H$ of $G$ there is a patterned left frame starter $S=\left\{\left(s_{i}, t_{i}\right)\right\}$ where $s_{i}^{-1}=t_{i}$ in $G-H$.

\section{Construction}

THEOREM 1. There exist frame starters in $Z_{2 n}-\{0, n\}$ if and only if $n \equiv 0$ or $1 \bmod 4$.

We prove this theorem by the following three lemmas and we use additive symbols.

LemMA 1. $Z_{8 k}-\{0,4 k\}$ has a frame starter for all $k \geqslant 1$.

Proof: For $k=1, Z_{8}-\{0,4\}$ has a frame starter $\left\{\left(\begin{array}{ll}1 & 3\end{array}\right),(25),(6 \quad 7)\right\}$. For $k>1$, let $S$ be the set of pairs of elements in $Z_{8 k}$ consisting of the following four collections:

(1) $(2 k-12 k+1),(2 k-32 k+3),(2 k-52 k+5), \ldots,(34 k-3)$, $(14 k-1)$;

(2) $(4 k-24 k+2),(4 k-44 k+4), \ldots,(2 k+46 k-4),(2 k+26 k-2)$;

(3) $(2 k 8 k-1),(6 k-18 k-2),(6 k 8 k-3),(6 k+18 k-4), \ldots$, $(7 k-27 k-1)$

(4) $(2 k-24 k+1),(2 k-44 k+3), \ldots,(46 k-5),(26 k-3)$;

We notice that the first pair in (3) is special. It is easy to see that the pairs in $S$ comprise all the nonzero elements except $4 k$. By direct calculation, the differences of 
the elements in each pair are

(1) $2,6,10, \ldots, 4 k-6,4 k-2,4 k+2,4 k+6, \ldots, 8 k-6,8 k-2$;

(2) $4,8, \ldots, 4 k-8,4 k-4,4 k+4,4 k+8, \ldots, 8 k-8,8 k-4$;

(3) $6 k-1,2 k-1,2 k-3, \ldots, 5,3,1,8 k-1,8 k-3, \ldots, 6 k+3,6 k+1,2 k+1$;

(4) $2 k+3,2 k+7,2 k+11, \ldots, 6 k-9,6 k-5,2 k+5,2 k+9, \ldots, 6 k-7,6 k-3$.

We can verify that these differences are all the elements in $Z_{8 k}-\{0,4 k\}$.

LEMMA 2. $Z_{16 k+2}-\{0,8 k+1\}$ has a frame starter for all $k \geqslant 1$.

ProOF: Let $S$ be the set of pairs of elements in $Z_{16 k+2}$ consisting of the following seven collections

(1) $(4 k 4 k+1),(4 k-14 k+2), \ldots,(2 k+26 k-1),(2 k+16 k)$;

(2) $(11 k+115 k+2),(11 k 15 k+3),(11 k-115 k+4), \ldots$, $(10 k+316 k),(10 k+216 k+1)$;

(3) $(k 7 k+1),(k-17 k+2), \ldots,(28 k-1),(18 k)$;

(4) $(12 k+214 k+2),(14 k+114 k+3),(14 k 14 k+4),(14 k-114 k+5)$, $\ldots,(13 k+415 k),(13 k+3,15 k+1)$;

(5) $(10 k+112 k+3),(10 k 12 k+4),(10 k-112 k+5), \ldots$, $(9 k+313 k+1),(9 k+213 k+2)$;

(6) $(7 k 11 k+2),(7 k-111 k+3), \ldots,(6 k+212 k),(6 k+112 k+1)$;

(7) $(2 k 8 k+2),(2 k-18 k+3), \ldots,(k+29 k),(k+19 k+1)$.

We notice that the first pair in (4) is special. We can see that the pairs in $S$ comprise all the nonzero elements in $Z_{16 k+2}$ except $8 k+1$. By direct calculation, the differences of the elements in every pair are

(1) $1,3,5, \ldots, 4 k-1,12 k+3,12 k+5, \ldots, 16 k-1,16 k+1$;

(2) $4 k+1,4 k+3, \ldots, 6 k-3,6 k-1,10 k+3,10 k+5, \ldots, 12 k-1,12 k+1$;

(3) $6 k+1,6 k+3, \ldots, 8 k-3,8 k-1,8 k+3,8 k+5, \ldots, 10 k-1,10 k+1$;

(4) $2 k, 2,4,6, \ldots, 2 k-4,2 k-2,14 k+4,14 k+6, \ldots, 16 k-2,16 k, 14 k+2$;

(5) $2 k+2,2 k+4, \ldots, 4 k-2,4 k, 12 k+2,12 k+4, \ldots, 14 k-2,14 k$;

(6) $4 k+2,4 k+4, \ldots, 6 k-2,6 k, 10 k+2,10 k+4, \ldots, 12 k-2,12 k$;

(7) $6 k+2,6 k+4, \ldots, 8 k-2,8 k, 8 k+2,8 k+4, \ldots, 10 k-2,10 k$.

It is easy to see these differences are all the elements in $Z_{16 k+2}-\{0,8 k+1\}$.

Lemma 3. $Z_{16 k+10}-\{0,8 k+5\}$ has a frame starter for all $k \geqslant 0$.

Proof: For $k=0, Z_{10}-\{0,5\}$ has a frame starter $\{(89),(13),(47),(26)\}$. For $k \geqslant 1$, let $S$ be the set of pairs of elements in $Z_{16 k+10}$ consisting of the following four collections

(1) $(4 k+14 k+3),(4 k-14 k+5),(4 k-34 k+7), \ldots,(38 k+1)$, $(18 k+3)$; 
(2) $(4 k+24 k+6),(4 k 4 k+8),(4 k-24 k+10), \ldots,(48 k+4)$, $(28 k+6)$;

(3) $(14 k+814 k+9),(10 k+714 k+10),(10 k+614 k+11)$, $(10 k+514 k+12), \ldots,(8 k+916 k+8),(8 k+816 k+9)$

(4) $(4 k+412 k+7),(8 k+712 k+8),(12 k+612 k+9)$, $(12 k+512 k+10), \ldots,(10 k+914 k+6),(10 k+814 k+7)$.

We notice that the first pair in (3) and the first two pairs in (4) are special. After these particular pairs, the first element in every pair decreases 1 while the second element in every pair increases 1 successively. It is easy to see that the elements in the pairs of $S$ comprise all the nonzero elements in $Z_{16 k+10}$ except $8 k+5$. Direct calculation shows that the differences of every pair in $S$ are

(1) $2,6,10, \ldots, 8 k-2,8 k+2,8 k+8,8 k+12,8 k+16, \ldots, 16 k+4,16 k+8$;

(2) $4,8,12, \ldots, 8 k, 8 k+4,8 k+6,8 k+10, \ldots, 16 k+2,16 k+6$;

(3) $1,4 k+3,4 k+5,4 k+7, \ldots, 8 k-1,8 k+1,8 k+9,8 k+11, \ldots, 12 k+5$, $12 k+7,16 k+9$

(4) $8 k+3,4 k+1,3,5,7, \ldots, 4 k-3,4 k-1,12 k+11,12 k+13, \ldots, 16 k+5$, $16 k+7,12 k+9,8 k+7$.

We can see that these differences comprise all the nonzero elements in $Z_{16 k+10}$ except $8 k+5$.

The dicycle group $Q_{2 n}$ of order $4 n$ is defined by

$$
Q_{2 n}=\left\langle\alpha, \beta: \alpha^{2 n}=e \beta^{2}=\alpha^{n}, \alpha \beta=\beta \alpha^{-1}\right\rangle .
$$

This is a nonabelian group with a unique element of order 2 .

THEOREM 2. $Q_{2 n}-\left\{e, \alpha^{n}\right\}$ has a left frame starter if and only if $n$ is a positive even integer.

Proof: Let $B$ be the complement of $\langle\alpha\rangle$ in $Q_{2 n}$. Every pair of elements in $Q_{2 n}$ has one of the following three types:

$$
\left(\alpha^{u} \beta \alpha^{x}\right), \quad\left(\alpha^{0} \alpha^{w}\right), \quad\left(\beta \alpha^{y} \beta \alpha^{z}\right) .
$$

If $S$ is a left frame starter of $Q_{2 n}-\left\{e, \alpha^{n}\right\}, S$ must have exactly $n$ pairs of the first type in order to yield $2 n$ elements of $B$. If $S$ has $j$ pairs of the second type, we have $n+2 j=2 n-2$. Hence $Q_{2 n}-\left\{e, \alpha^{n}\right\}$ has no left frame starters if $n$ is odd.

From now on assume that $n=2 k$. Let $S$ be the set of pairs of elements in $Q_{2 n}$ consisting of the following three collections:

(1) $\left(\alpha^{k} \beta \alpha^{4 k-1}\right),\left(\alpha^{2 k+1} \beta \alpha^{2 k-2}\right),\left(\alpha^{2 k+2} \beta \alpha^{2 k-4}\right),\left(\alpha^{2 k+3} \beta \alpha^{2 k-6}\right), \ldots$, $\left(\alpha^{3 k} \beta\right),\left(\alpha^{3 k+1} \beta \alpha^{2 k-3}\right),\left(\alpha^{3 k+2} \beta \alpha^{2 k-5}\right), \ldots,\left(\alpha^{4 k-2} \beta \alpha^{3}\right)$, $\left(\alpha^{4 k-1} \beta \alpha\right)$ 
(2) $\left(\alpha \alpha^{2 k-1}\right),\left(\alpha^{2} \alpha^{2 k-2}\right),\left(\alpha^{3} \alpha^{2 k-3}\right), \ldots,\left(\alpha^{k-1} \alpha^{k+1}\right)$

(3) $\left(\beta \alpha^{2 k-1} \beta \alpha^{4 k-2}\right),\left(\beta \alpha^{2 k} \beta \alpha^{4 k-3}\right),\left(\beta \alpha^{2 k+1} \beta \alpha^{4 k-4}\right), \ldots$, $\left(\beta \alpha^{3 k-3} \beta \alpha^{3 k}\right),\left(\beta \alpha^{3 k-2} \beta \alpha^{3 k-1}\right)$.

It is easy to see that these pairs contain all the nonidentity elements in $Q_{2 n}$ except $\alpha^{n}$. We notice that the first pair in (1) is special. The elements $s_{i}^{-1} t_{i}$ and $t_{i}^{-1} s_{i}$, where $\left(s_{i}, t_{i}\right)$ varies through $S$, are the following

(1) $\beta \alpha^{k-1}, \beta \alpha^{4 k-1}, \beta \alpha^{4 k-2}, \beta \alpha^{4 k-3}, \ldots, \beta \alpha^{3 k}, \beta \alpha^{k-2}, \beta \alpha^{k-3}, \ldots, \beta \alpha$, $\beta, \beta \alpha^{2 k}, \beta \alpha^{2 k+1}, \ldots, \beta \alpha^{3 k-3}, \beta \alpha^{3 k-2}, \beta \alpha^{k}, \beta \alpha^{k+1}, \ldots, \beta \alpha^{2 k-1}$, $\beta \alpha^{3 k-1}$

(2) $\alpha^{2 k-2}, \alpha^{2 k-4}, \alpha^{2 k-6}, \ldots, \alpha^{2}, \alpha^{4 k-2}, \alpha^{4 k-4}, \ldots, \alpha^{2 k+4}, \alpha^{2 k+2}$;

(3) $\alpha^{2 k-1}, \alpha^{2 k-3}, \alpha^{2 k-5}, \ldots, \alpha^{3}, \alpha, \alpha^{4 k-1}, \alpha^{4 k-3}, \ldots, \alpha^{2 k+3}, \alpha^{2 k+1}$.

These elements are all the elements in $Q_{2 n}-\left\{e, \alpha^{n}\right\}$, and therefore $S$ is a left starter of $Q_{2 n}-\left\{e, \alpha^{n}\right\}$.

\section{REFERENCES}

[1] J.H. Dinitz and D.R. Stinson, 'The construction and uses of frames', Ars Combin. 10 (1980), 31-53.

[2] D.F. Hsu and A.D. Keedwell, 'Generalized complete mappings, neofields, sequenceable groups and block designs I', Pacific J. Math. 111 (1984), 317-332.

[3] P.A. Leonard, 'Adders for the patterned starter in some nonabelian groups', Bull. Austral. Math. Soc. 10 (1974), 321-323.

[4] C-D. Wang and A.D. Keedwell, 'On special near orthomorphisms', J. Combin. Math. Combin. Comput. (to appear).

Department of Mathematics

Beijing Institute of Technology

Xicheng Beijing

100031 China 